\title{
The "Sigma Test": a new methodology for evaluating a tennis player
}

\author{
Salvatore Buzzelli
}

\section{ABSTRACT}

This article aims to illustrate an innovative and absolutely functional method of assessing the fitness of a tennis player, based on the registration of an incremental metabolic effort, under attentive aegis.

\author{
Key words: sigma test, tennis, \\ assessment test, physical form, \\ attention energy cost. \\ Received: 15 May 2020 \\ Accepted: 18 September 2020 \\ Corresponding author: \\ Salvatore Buzzelli, Italy. Email: \\ salvatorebuzzelli1@gmail.com
}

\section{INTRODUCTION}

In the functional evaluation of the tennis player, especially when one wants to investigate his Maximum Power Aerobics (VO2max), it is customary to resort to evaluation tests already widely known and equipped with a considerable amount of scientific and methodological documentation.

The most used tests in this field range from the Cooper test (12 minutes of continuous and linear running) (Fox, 1973); to the Leger test (increasing levels of running rhythms every minute, in linear running and changes of direction every $20 \mathrm{~m}$, until exhaustion) (Léger,1988); to the YoYo Test (linear runs of $20 \mathrm{~m}$ with change of direction, at increasing rates guided by an audio track, until exhaustion) (Krustrup, 2003).

These tests, although predictive of the Maximum Aerobic Performance Capacity, have a structural and methodological limit of great importance in a sport such as Tennis, in fact in their execution they run in non-specific mode, always over distances not suitable for tennis movements and above all not account is taken of the attentional aspect, which plays a decisive role in competitive performance (Tamorri, 2000; Buzzelli, 2007; Smith, 2016).

In fact, unlike other athletes of other situation sports, the tennis player moves over short distances ( $80 \%$ of the movements are within a radius of $4-5 \mathrm{~m}$ ) in the direction of the incoming ball and will have to raise attention levels if he wishes respect the right times to hit the best and in the best position.
Starting from these last fundamental considerations, in 2007, a specific test (Sigma Test) (Buzzelli, 2007), was developed that would respond in a more functional way to what you really

needed to know, that is what is the maximum capacity of organic resistance in an attentive form, on which develop very specific training plans.

\section{DESCRIPTION}

The "Sigma Test" is a test designed and developed to investigate the specific resistance of a tennis player. The development of the test was inspired by multistage fitness test, such as the Legér test or the YoYo test, but unlike these tests, the participants are running shuttles on 5,5 meters, repeated until the physical and mental abilities of reaction and specific stroke, are exhausted.

It is performed in one half of the tennis court and both metabolic and attentional aspects are involved in its realization. It is guided by an electronic track based on visual and acoustic signals. It is possible to carry out 2 types of sigma test; one with only acoustic trace (default, non-attentional), the other with acoustic and visual trace (original, attentive). The difference in terms of the results of the two tests, will provide us with the "Energy Cost of Attention" (Buzzelli, 2014). However, we will not deal with this possibility in this article which we may defer to another occasion.

\section{MATERIALS AND METHODS}

To carry out this test, starting from 2007 (Buzzelli, 2007), a special tool called initially "SensoTouch" and then definitively "SensoBuzz", was used but currently it is possible to use a smartphone application, also called "SensoBuzz" (Buzzelli, 2019), 
which emits visual and acoustic signals in a random mode with a pre-established time scan.

The exercise is performed on one half of the tennis court organized as shown in figure 1 . The correct execution of the test involves shuttle movements, in which the chaining of the runstop-restart action towards and from a target corresponding to the signal emitted must be correctly managed, starting from a point called "base" and avoiding remains still on it, waiting for the next signal.

The introductory minute is just to allow the student to correctly adapt the speed of movement to the rhythm of the signals. Each minute the "SensoBuzz" decreases the emission rhythm by 0.2 seconds, causing the movement speed to be increased. Each signal corresponds to a complete round trip of 11 meters, that is 5.50 meters to go and as many to return to the "base". The instrument counts the number of "shuttles" and the total working time.

The errors of moving to a target other than the one indicated, are allowed and will be recorded by the examiner during the execution of the test. It is also possible that the student sees the error and corrects himself by heading in the right direction. Of course, errors cause uncertainty and hesitancy in moving, requiring the student to take small shots to recover space and remain in the rhythm of the test.

At the end of the test, a large number of errors will have resulted in a supplementary effort determined by the sum of the accelerations, with the consequence of a reduction in the overal performance capacity. The test ends when the athlete is still in the vicinity of a finish line while a new signal is emitted, therefore in considerable delay that he can no longer fill with any running accelerations.

The following are recorded: the number of shuttle runs performed correctly (corresponding to the number shown on the instrument display) as well as the chronological calculation of any errors made during the whole test.

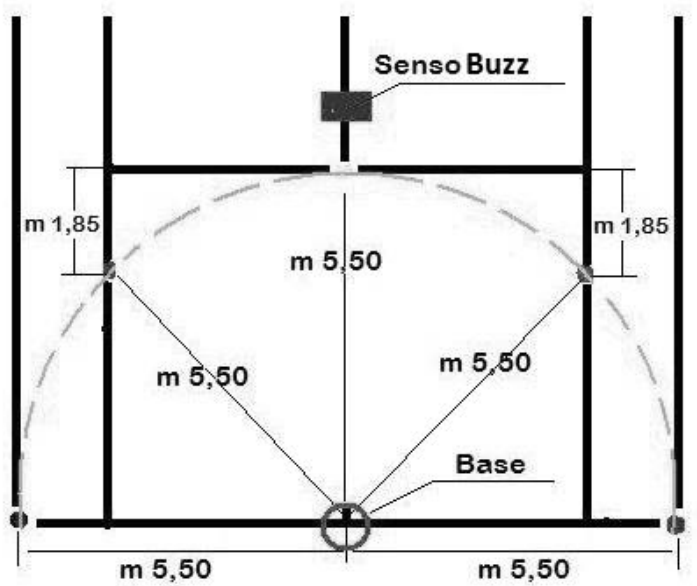

Figure 1. Test set up on-court.
The test, which is carried out by carrying out specific stroke movements, also involves the ability to pay attention and maintain concentration. The examinee, following the rhythm imposed by "SensoBuzz", must move with shuttle runs (round trip) from a central point called "base" to a goal called "target" and return. The return to the "base" must correspond to the reception of a new signal issued by "SensoBuzz".

The targets are five, and are placed in a semicircle at a distance of 5.50 meters radially from the "base" and placed randomly in the fixed points of the portion of the tennis court used, as shown in figure 1. The targets are associated with five different signals, which by convention have been set in three visual (red, blue, yellow) and two audible: one acute and one double.

The test is incremental starting from a rhythm of signal emission one every 5 seconds; this rhythm allows the examinee to adapt to the test, being able to move quite easily and enter the test rhythm, during the first minute. At each minute, the emission time between one signal and the other (split time) decreases by 0.2 seconds, with consequent adjustment of the movement speed by the examining member.

\section{TEST PROTOCOL}

The examining member places himself in a standing position on the "base" ready to activate at the first signal. When the first signal is emitted, he will run towards the corresponding target, touch the space immediately facing the "goal" with his foot and with a decisive change of direction without turning his back on the midfield network, he returns to the "base", preparing to perform the shift relative to the next signal.

The movements must follow the specific running technique, as if you were playing tennis, according to the rhythm defined by the instrument. To have a reliable assessment of the organic resistance, the test must be carried out for at least 3 minutes. The test ends when the examining member's inability to sustain the rhythm imposed by the instrument will be manifested, precisely when the student is on a target and the instrument emits another signal.

When this event occurs, the "SensoBuzz" application will be stopped by the operator and the total duration of the test, the number of signals performed, the meters travelled (according to the formula Meters $=$ Cycles $\times 11$ ) will automatically be highlighted in a screen., where 11 represents the base-target-base route expressed in meters), the specific speed reached in the test in $\mathrm{Km} / \mathrm{h}$ and $\mathrm{m} / \mathrm{s}$ and the theoretical value of the Maximum Oxygen Consumption according to the formula:

VO2max $=33,3+(3,3 *$ Speed $)-(3,25 *$ Age $)+((0,2 *$ Speed $) *$ Age), (Lèger, 1988)

With these data available, the coach can then suggest personalized training according to the motor and metabolic capacity he wants to develop in the athlete. The data shown in Table 1, show the values corresponding to the result expressed in cycles, obtained in the test. 
Table 1. Sigma

Test

Values.

\begin{tabular}{|c|c|c|c|c|c|c|}
\hline $\begin{array}{c}\text { Duration of } \\
\text { the Test }\end{array}$ & $\begin{array}{l}\text { Test } \\
\text { step }\end{array}$ & $\begin{array}{l}\text { Interval between } \\
\text { signals (sec.) }\end{array}$ & $\begin{array}{l}\text { Total } n^{\circ} \\
\text { signals }\end{array}$ & $\begin{array}{l}\text { Total meters } \\
\text { paths }\end{array}$ & \begin{tabular}{|c|} 
Average speed \\
$(\mathrm{Km} / \mathrm{h})$
\end{tabular} & $\begin{array}{c}\text { VO2 } \max \\
\text { (ml/Kg/min) } \\
\text { (theoretical) } \\
\text { (average values for ages } \\
\text { between } 17-19 \text { years) }\end{array}$ \\
\hline $0^{\prime} 00^{\prime \prime}$ & --- & 5 & --- & --- & --- & --- \\
\hline $0^{\prime} 30^{\prime \prime}$ & 0,5 & $"$ & 6 & 66 & 7,92 & --- \\
\hline $1^{\prime} 00^{\prime \prime}$ & 1 & 4,8 & 12 & 132 & & 29,86 \\
\hline $1^{\prime} 30^{\prime \prime}$ & 1,5 & $"$ & 18 & 200 & 8,25 & 30,43 \\
\hline $2^{\prime} 00^{\prime \prime}$ & 2 & 4,6 & 24 & 269 & & 31,68 \\
\hline $2^{\prime} 30^{\prime \prime}$ & 2,5 & $"$ & 30 & 341 & 8,58 & 32,23 \\
\hline $3^{\prime} 00^{\prime \prime}$ & 3 & 4,4 & 37 & 412 & & 33,51 \\
\hline $3^{\prime} 30^{\prime \prime}$ & 3,5 & $"$ & 44 & 487 & 8,91 & 35,35 \\
\hline $4^{\prime} 00^{\prime \prime}$ & 4 & 4,2 & 51 & 561 & & 37,17 \\
\hline $4^{\prime} 30^{\prime \prime}$ & 4,5 & $"$ & 58 & 638 & 9,24 & 38,22 \\
\hline $5^{\prime} 00^{\prime \prime}$ & 5 & 4,0 & 65 & 715 & & 40,83 \\
\hline $5^{\prime} 30^{\prime \prime}$ & 5,5 & " & 72 & 797 & 9,9 & 41,12 \\
\hline $6^{\prime} 00^{\prime \prime}$ & 6 & 3,8 & 80 & 880 & & 42,66 \\
\hline $6^{\prime} 30^{\prime \prime}$ & 6,5 & " & 88 & 978 & 10,56 & 44,71 \\
\hline $7^{\prime} 00^{\prime \prime}$ & 7 & 3,6 & 96 & 1056 & & 46,32 \\
\hline 7'30" & 7,5 & $"$ & 104 & 1147 & 10,89 & 47,83 \\
\hline $8^{\prime} 00^{\prime \prime}$ & 8 & 3,4 & 112 & 1237 & & 49,97 \\
\hline $8^{\prime} 30^{\prime \prime}$ & 8,5 & " & 121 & 1333 & 11,55 & 52,54 \\
\hline $9^{\prime} 00^{\prime \prime}$ & 9 & 3,2 & 130 & 1430 & & 55,46 \\
\hline $9^{\prime} 30^{\prime \prime}$ & 9,5 & " & 139 & 1532 & 12,21 & 57,85 \\
\hline $10^{\prime} 00^{\prime \prime}$ & 10 & 3,0 & 148 & 1633 & & 60,95 \\
\hline $10^{\prime} 30^{\prime \prime}$ & 10,5 & $"$ & 158 & 1743 & 13,20 & 63,07 \\
\hline $11^{\prime} 00^{\prime \prime}$ & 11 & 2,8 & 168 & 1853 & & 66,43 \\
\hline $11^{\prime} 30^{\prime \prime}$ & 11,5 & $"$ & 181 & 1972 & 14,19 & 69,34 \\
\hline $12^{\prime} 00^{\prime \prime}$ & 12 & 2,6 & 190 & 2090 & & 73,75 \\
\hline $12^{\prime} 30^{\prime \prime}$ & 12,5 & " & 202 & 2216 & 15,18 & 76,03 \\
\hline $13^{\prime} 00^{\prime \prime}$ & 13 & 2,4 & 213 & 2343 & & 78,45 \\
\hline
\end{tabular}


The following are the average values of the cycles performed and Dev.St. divided by age, relating to the two genders (male and female).

\begin{tabular}{|l|l|l|l|l|l|}
\hline Age & $11-12$ & $13-14$ & $15-16$ & $17-18$ & $\begin{array}{l}19 \text { and } \\
\text { more }\end{array}$ \\
\hline Male & 56,9 & 76,3 & 94,1 & 116,7 & 124,6 \\
\hline Dev.St. & 21,8 & 14,2 & 17,3 & 10,6 & 11,3 \\
\hline Female & 59,2 & 70,2 & 83,4 & 92,3 & 97,2 \\
\hline Dev.St. & 10,4 & 24,6 & 16,3 & 19,4 & 14,1 \\
& & & \multicolumn{3}{|c|}{$(p<0,05)$} \\
\hline
\end{tabular}

Table 2. "Sigma Test" and age: average reference values of cycles.

\section{CONCLUSIONS}

The "Sigma test" is a simple test even for very young athletes (at least 10 years of age). It is performed on a portion of the tennis court and consequently does not need special measurements, provided that the court is approved. Alternatively, it can be performed in a space delimited by a semicircle with a radius of 5.5 meters from the centre, on which 5 targets are arranged randomly which must be positioned as the cardinal points North, East, West, North-East and North-West.

Data processing takes place automatically and shown to the user, in a final screen after stopping the test, from the "SensoBuzz" application. A fit tennis player performs a large number of shuttles, recording none or very few sequence errors.

\section{REFERENCES}

Buzzelli, S (2007). SensoTouch Buzzelli System, Pubblicazioni, available at: www.salvatorebuzzelli.it.

Buzzelli, S. (2013). SensoBuzz, Pubblicazioni, C.E.Youcanprint.

Buzzelli, S. (2014). Costo energetico dell'attenzione, Ricerca Scientifica, available at www.salvatorebuzzelli.it.

Fox, E. L. (1973). A simple accurate technique for predicting maximal aerobic power. J Appl Physiol.5: 914-6, https://doi.org/10.1152/jappl.1973.35.6.914

Krustrup, P., Mohr, M., Amstrup, T., Rysgaard, T., Johansen, J., Steensberg, A., ... \& Bangsbo, J. (2003). The yo-yo intermittent recovery test: physiological response, reliability, and validity. Medicine \& Science in Sports \& Exercise, 35(4), 697-705, https://doi.org/10.1249/01.MSS.0000058441.94520.32

Leger, L. A., Mercier, D., Gadoury, C., \& Lambert, J. (1988). The multistage 20 metre shuttle run test for aerobic fitness. Journal of Sports Sciences, 6(2), 93-101, https://doi.org/10.1080/02640418808729800

Smith, M. R., Zeuwts, L., Lenoir, M., Hens, N., De Jong, L. M., \& Coutts, A. J. (2016). Mental fatigue impairs soccer-specific decision-making skill. Journal of Sports Sciences, 34(14), 1297-1304, https://doi.org/10.1080/02640414.2016.1156241

Tamorri, S. (2000). Neuroscienze e sport: psicologia dello sport, processi mentali dell'atleta, Utet.
RECOMMENDED ITF TENNIS ACADEMY CONTENT (CUCK BELOW)

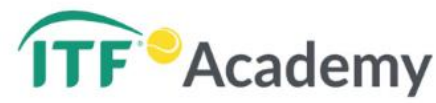

Copyright (c) 2020 Salvatore Buzzelli

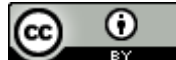

This text is under a Creative Commons BY 4.0 license

You are free to Share - copy and redistribute the material in any medium or format - and Adapt the content - remix, transform, and build upon the material for any purpose, even commercially under the following terms:

Attribution: You must give appropriate credit, provide a link to the license, and indicate if changes were made. You may do so in any reasonable manner, but not in any way that suggests the licensor endorses you or your use.

CCBY4.0 license terms summary $\quad$ CCBY4.0license terms 